biblio.ugent.be

The UGent Institutional Repository is the electronic archiving and dissemination platform for all UGent research publications. Ghent University has implemented a mandate stipulating that all academic publications of UGent researchers should be deposited and archived in this repository. Except for items where current copyright restrictions apply, these papers are available in Open Access.

This item is the archived peer-reviewed author-version of:

Two special classes of space-times admitting a non-null valence two Killing spinor N. Van den Bergh

In: Classical and Quantum Gravity, 27, 015004, 2010.

To refer to or to cite this work, please use the citation to the published version:

N. Van den Bergh (2010). Two special classes of space-times admitting a non-null valence two Killing spinor. Classical and Quantum Gravity, 27, 1-11.

doi:10.1088/0264-9381/27/1/015004 


\title{
Two special classes of space-times admitting a non-null valence two Killing spinor
}

\author{
N. Van den Bergh \\ Ghent University, Department of Mathematical Analysis IW16, \\ Galglaan 2, 9000 Ghent, Belgium \\ E-mail: norbert .vandenbergh@ugent . be
}

\begin{abstract}
Non-conformally flat space-times admitting a non-null Killing spinor of valence two are investigated in the Geroch-Held-Penrose formalism. Contrary to popular belief these space-times are not all explicitly known. It is shown that the standard construction hinges on the tacit assumption that certain integrability conditions hold, implying two algebraic relations, $K S_{1}$ and $K S_{2}$, for the spin coefficients and the components of the Ricci spinor. An exhaustive list of (conformal classes of) space-times, in which either $K S_{1}$ or $K S_{2}$ are violated, is presented. The resulting space-times are each other's Sachs transforms, in general admit no Killing vectors and are characterized by a single arbitrary function.
\end{abstract}

PACS numbers: 04.20.Jb

\section{Introduction}

The concept of a Killing spinor has its origins in the work of Walker and Penrose [31] who, in the class $\mathcal{D}_{0}$ of Petrov type D solutions of Einstein's vacuum field equations with cosmological constant, demonstrated the existence of a valence two symmetric spinor $X_{A B}$, satisfying the conformally invariant (twistor) equation

$$
\nabla_{A^{\prime}(A} X_{B C)}=0 .
$$

The original significance of such a spinor is that its existence in a space-time $(\mathcal{M}, \mathrm{g})$ determines a constant of the motion along any null geodesic. The existence of this constant of the motion in $\mathcal{D}_{0}$ may be equivalently derived from the separability of the Hamilton-Jacobi equation for the null geodesics therein [5]. Introducing the two-form $D_{a b}=X_{A B} \epsilon_{A^{\prime} B^{\prime}}+\bar{X}_{A^{\prime} B^{\prime}} \epsilon_{A B}$, called a conformal Killing-Yano (CKY) tensor, the equation corresponding to $(1)$ reads $[12,29]$

$$
\nabla_{(a} D_{b) c}=\frac{1}{3} \nabla_{d}\left(g_{a b} D_{c}^{d}-D_{(a}^{d} g_{b) c}\right),
$$

or, equivalently [1],

$$
3 \nabla_{c} D_{a b}=3 \nabla_{[c} D_{a b]}-2 \nabla_{d} D_{[a}^{d} g_{b] c} .
$$


As a consequence these space-times are also frequently called CKY space-times [10]. When the right hand side of (2) vanishes, $D_{a b}$ is called a Killing-Yano tensor or a Penrose-Floyd tensor [25]. This happens if and only if $X_{A B}$ satisfies the additional skew Hermiticity condition

$$
\nabla_{A^{\prime}}^{B} X_{A B}+\nabla_{A}^{B^{\prime}} \bar{X}_{A^{\prime} B^{\prime}}=0 .
$$

A space-time admitting a valence two Killing-Yano tensor will be called a KY space-time: all these have been determined explicitly [7, 8, 18, 25]. Note that a CKY space-time is not necessarily conformally related to a KY space-time, as examples in [14] and [20] show $\ddagger$. The square $K_{a b}=D_{a}{ }^{c} D_{c b}$ of a KY tensor $D$ is a Killing tensor (KT), namely a symmetric tensor satisfying the equation

$$
\nabla_{(a} K_{b c)}=0
$$

and giving rise to a quadratic first integral of the geodesic equation. Similarly the square of a CKY tensor is a conformal Killing tensor (CKT), namely a symmetric tensor satisfying

$$
\nabla_{(a} K_{b c)}=g_{(a b} k_{c)}
$$

where $k_{c}$ is obtained by contraction of (6) with $g^{a b}$. Conformal Killing tensors are of interest inasmuch as they give rise to quadratic first integrals for null geodesics. For the sequel it is worth noting that, if $K_{a b}$ is a conformal Killing tensor, then so is $K_{a b}+f g_{a b}$ with $f$ an arbitrary function (both defining the same first integrals of the null geodesic equation). This implies that one can always assume $K_{a b}$ to be traceless, implying $k_{c}=\frac{1}{3} \nabla_{m} K^{m}{ }_{c}$. Note that the converse of the property above not necessarily holds: not every (conformal) Killing tensor is the square of a (conformal) Killing-Yano tensor and conditions which a $(\mathrm{C}) \mathrm{KT}$ space-time has to obey in order to be a $(\mathrm{C}) \mathrm{KY}$ space-time have been discussed in [4] and [10]. These conditions are satisfied for example in those subclasses of the type D vacuum solutions and the aligned non-null Einstein-Maxwell solutions that admit a Killing tensor $[17,4,28]$. In $[3,11]$ it was furthermore shown that every type D CKT space-time with a Killing tensor of Segre type $[(11),(11)]$ is CKY.

The Weyl spinor of a non-conformally flat space-time admitting a non-null valence two Killing spinor $X_{A B}$ is necessarily of Petrov type $\mathrm{D}$ with repeated principal spinors aligned with the principal spinors of $X_{A B}$ [27], while the Weyl principal null directions define geodesic shear-free $\S$ null congruences. Henceforth I will call these KS space-times, to distinguish them from the more general CKY space-times, for which the Petrov type also can be $\mathrm{N}$ or O. Obviously, as (1) is conformally invariant, KS space-times can only be determined up to an arbitrary conformal transformation of the metric. KS

$\ddagger$ to add to the confusion spinors satisfying (1) were termed conformal Killing spinors in [2] and [5], while the name Killing spinor or Killing spin two-forms was reserved for spinors or their tensor analogues satifying also (4). As explained in [14] it is better to refrain from this terminology.

$\S$ this property also holds $[9,15]$ for the two null eigendirections of any (conformal) Killing tensor of Segre type $[(11),(11)])$. 
space-times necessarily include all those which are conformally related to the Petrov type D KY space-times. The inclusion is strict: examples exist which satisfy (1) but not (4): they appear in the classes $\mathcal{D}$ and $\mathcal{D}_{0}$ of solutions of Einstein's electrovac and vacuum field equations with cosmological constant for a non-null aligned Maxwell field as the Kinnersley Case III solutions [5, 6]. A KS space-time always admits a conformal representant in which the trace of the associated conformal Killing tensor is constant. In this representant the conformal Killing tensor becomes a Killing tensor, but, by construction, has two constant eigenvalues (the reason why this is so will become clear in section 2). As the non-constancy of the eigenvalues plays an essential role in the construction of appropriate coordinates $[15,14,23,24]$ and in the ensuing separability properties, one may ask whether a conformal transformation exists which preserves the existence of the Killing tensor and which leads to non-constant eigenvalues (the so-called non-singular Killing tensors [16]). In [14] the answer to this question was taken to be affirmative, as a consequence of the tacit assumption of two integrability conditions, introduced in section 2 below as $K S_{1}$ and $K S_{2}$. The resulting list of canonical lineelements of KS space-times therefore turned out to be incomplete. This was partially remedied in [20], where the extra line-elements were constructed which arise in the 'I-N family' of [14], defined by the vanishing of one of the spin coefficients $\rho$ or $\mu$. In the present paper the extra line-elements are constructed which arise in the general 'family I', defined by the non-vanishing of $\rho, \mu, \pi$ and $\tau$, and by assuming that only one of the two integrability conditions $K S_{1}$ or $K S_{2}$ holds. In order to fully exploit the symmetry provided by the alignment of the principal null directions of the Weyl and Killing spinor, the Geroch-Held-Penrose formalism [13] is used throughout: the $K S_{1}$ and $K S_{2}$ families are then each other's Sachs transforms [13].

\section{Preliminaries}

Writing the Killing spinor as $X_{A B}=X o_{(A} \iota_{B}$ and using $o, \iota$ as the basis spinors for the GHP formalism, the components of (1) imply (see [14] for details)

$$
\kappa=\sigma=0
$$

and

$$
\begin{aligned}
& \mathrm{P} X=-\rho X, \\
& \mathrm{\partial} X=-\tau X,
\end{aligned}
$$

together with their 'primed versions' and $X^{\prime}=X$, namely $\kappa^{\prime}=\sigma^{\prime}=0$ and $\mathrm{P}^{\prime} X=-\rho^{\prime} X$, $\partial^{\prime} X=-\tau^{\prime} X$.

From (7), the application of the [ð, $\mathrm{P}]$ commutator to $X$ and the GHP equations one finds that the principal null directions of the Killing spinor and the Weyl spinor are aligned, i.e. $\Psi_{2}$ is the only non-vanishing component of the Weyl spinor. The equations $(7,8,9)$ are invariant under conformal transformations $g \rightarrow \Omega^{2} g, X \rightarrow \Omega X$ 
and a conformal representant $(\mathcal{M}, \hat{g})$ can be fixed by imposing $|\hat{X}|=1$. In the manifold $(\mathcal{M}, \hat{g})$ we have then

$$
\hat{\rho}+\overline{\hat{\rho}}=\hat{\rho}^{\prime}+\overline{\hat{\rho}}^{\prime}=\hat{\tau}+\overline{\hat{\tau}}^{\prime}=0,
$$

while in the $(\mathcal{M}, g)$ manifold with $g=\Omega^{2} \hat{g}$ one has $\Omega^{2}=X \bar{X}$. The cases $\tau=\tau^{\prime}=0$ and $\rho$ or $\rho^{\prime}=0$ have been discussed in [14] and [20], so henceforth $\rho, \rho^{\prime}, \tau, \tau^{\prime}$ will be taken $\neq 0$.

Defining the trace-free tensor $P_{a b}=X_{A B} \bar{X}_{A^{\prime} B^{\prime}}$, or, with null vectors $\ell_{a}=o_{A} o_{A^{\prime}}$, $n_{a}=\iota_{A} \iota_{A^{\prime}}$ and $m_{a}=o_{A} \bar{\iota}_{A^{\prime}}$,

$$
P_{a b}=\frac{\Omega^{2}}{2}\left(m_{(a} \bar{m}_{b)}+\ell_{(a} n_{b)}\right),
$$

one sees that $P_{a b}$ obeys the CKT equation (6). This implies that $P_{a b}$ will be the tracefree part of a Killing tensor $K_{a b}$, provided a solution $K_{a b}=P_{a b}+\frac{1}{4} K g_{a b}$ exists of the KT equation (5). Contraction implies the necessary and sufficient condition

$$
\nabla_{a} P_{c}^{a}+\frac{3}{4} \nabla_{c} K=0 .
$$

In components $\|$ this becomes

$$
\begin{aligned}
& \mathrm{\textrm { }} K-\Omega^{2}(\rho+\bar{\rho})=0, \\
& \supset K+\Omega^{2}\left(\tau+\bar{\tau}^{\prime}\right)=0 .
\end{aligned}
$$

or, in terms of the eigenvalues $a=\left(\Omega^{2}-K\right) / 4$ and $b=\left(\Omega^{2}+K\right) / 4$ of

$$
\begin{aligned}
& K_{c d}=2 a m_{(c} \bar{m}_{d)}+2 b \ell_{(c} n_{d)}: \\
& \mathrm{P} b=0, \\
& \mathrm{\partial} a=0 .
\end{aligned}
$$

By $(8,9)$ and $\Omega^{2}=X \bar{X}$ this also implies

$$
\begin{aligned}
& \mathrm{P} a=-(a+b)(\rho+\bar{\rho}), \\
& \precsim b=-(a+b)\left(\tau^{\prime}+\bar{\tau}\right),
\end{aligned}
$$

such that $(16,17,18,19)$ alternatively can be written as

$$
\begin{aligned}
\mathrm{d} a & =-(a+b)\left[(\rho+\bar{\rho}) \boldsymbol{\omega}^{4}+\left(\rho^{\prime}+\bar{\rho}^{\prime}\right) \boldsymbol{\omega}^{3}\right] \\
\mathrm{d} b & =-(a+b)\left[\left(\tau+\bar{\tau}^{\prime}\right) \boldsymbol{\omega}^{1}+\left(\bar{\tau}+\tau^{\prime}\right) \boldsymbol{\omega}^{2}\right],
\end{aligned}
$$

which exhibits clearly the relevance of the existence of a Killing tensor with non-constant eigenvalues for the construction of appropriate coordinates. It also becomes obvious now, as mentioned in the introduction, that all KS space-times admit at least one conformal representant (namely $(\mathcal{M}, \hat{g})$ or its constant re-scalings) in which a Killing $\|$ there is a sign difference with [14], which is probably due to the use of a different signature convention; I use $\ell^{a} n_{a}=1=-m^{a} \bar{m}_{a}$. The correspondence with the Newman-Penrose operators and the basis oneforms is taken as $\left(m^{a}, \bar{m}^{a}, n^{a}, \ell^{a}\right) \leftrightarrow(\delta, \bar{\delta}, \Delta, D)$ and $\left(-m_{a},-\bar{m}_{a}, \ell_{a}, n_{a}\right) \leftrightarrow\left(\boldsymbol{\omega}^{1}, \boldsymbol{\omega}^{2}, \boldsymbol{\omega}^{3}, \boldsymbol{\omega}^{4}\right)$ 
tensor exists, but that by (10) this particular Killing tensor has constant eigenvalues. If one assumes that there is a non-trivial conformal representant in which $a$ and $b$ are not both constants, i.e. if one assumes that non-constant solutions exist of the system $(16,17)$ or $(20,21)$ then extra integrability conditions result (namely $\mathrm{dd} a=\mathrm{dd} b=0$ ). It is preferable to study these equations in the $(\mathcal{M}, \hat{g})$ manifold, where the symbol from here onwards will be dropped: the remaining spin coefficients are then $\rho, \rho^{\prime}$ and $\tau=-\bar{\tau}^{\prime}$. Under the conditions (10) the integrability conditions for the system $(8,9)$ simplify to

$$
\begin{aligned}
& \mathrm{P}^{\prime} \rho-\mathrm{P} \rho^{\prime}=0, \\
& \check{\partial} \tau^{\prime}-\mathrm{\partial}^{\prime} \tau=0, \\
& \mathrm{P} \tau^{\prime}-\mathrm{\partial}^{\prime} \rho=0 .
\end{aligned}
$$

The GHP equations, together with the directional derivatives of (10), reduce to the system

$$
\begin{aligned}
& \mathrm{P} \rho=0, \\
& \mathrm{\partial} \rho=2 \rho \tau+\Phi_{01}, \\
& \mathrm{P} \tau=2 \rho \tau+\Phi_{01}, \\
& \mathrm{\partial} \tau=0, \\
& \mathrm{P} \rho^{\prime}-\check{\partial} \tau^{\prime}=-\rho \rho^{\prime}-\tau \bar{\tau}-\Psi_{2}-\frac{1}{12} R
\end{aligned}
$$

and impose the following restrictions on the curvature:

$$
\Phi_{00}=-\rho^{2}, \Phi_{02}=-\tau^{2} .
$$

All these equations are to be considered as being accompanied by their complex conjugates as well as their 'primed versions' (taking into account $\Phi_{00}^{\prime}=\Phi_{22}, \Phi_{02}^{\prime}=\Phi_{20}$, $\Phi_{10}^{\prime}=\Phi_{12}$ and (10)). The remaining derivatives of the spin coefficients are related by $(22,23)$, while $(24)$ becomes an identity under $\left(\overline{26}, 27^{\prime}\right)$. Also (23) and (29) imply that the real part of

$$
\Psi_{2}=E+i H
$$

can be written as

$$
E=-\frac{R}{12}-\rho \rho^{\prime}+\tau \tau^{\prime}
$$

It is advantageous now to introduce 0-weighted quantities $u, v$ (real and with $u^{\prime}=u$, $v^{\prime}=v$ ) and $\phi, \phi^{\prime}$ (complex) for the remaining curvature components as follows:

$$
\begin{aligned}
& R=8(u-v)-16 \rho \rho^{\prime}, \\
& \Phi_{11}=u+v-2 \rho \rho^{\prime}, \\
& \Phi_{01}=-3 \rho \tau-2 \frac{\rho}{\tau^{\prime}} \phi .
\end{aligned}
$$

Under the ' operation this also implies $\Phi_{21}=-3 \rho^{\prime} \tau^{\prime}-2 \rho^{\prime} \phi^{\prime} / \tau$.

The integrability conditions for the system $(16,17)$ become then

$$
\rho^{\prime} \mathrm{P} a-\rho \mathrm{P}^{\prime} a=0
$$


and

$$
\tau^{\prime} \partial b-\tau ð^{\prime} b=0
$$

Acting respectively with $\partial$ and $\mathrm{P}, \mathrm{P}^{\prime}$ on (36) and (37) and eliminating the second order derivatives by means of the $\left[\partial^{\left({ }^{\prime}\right)}, \mathrm{P}^{\left({ }^{\prime}\right)}\right]$ commutator relations, results in two more conditions,

$$
\rho^{\prime} \bar{\phi}^{\prime} \mathrm{P} a+\rho \phi \mathrm{P}^{\prime} a=0
$$

and

$$
\tau^{\prime} \bar{\phi} \precsim b+\tau \phi \check{\partial}^{\prime} b=0 .
$$

All further derivatives being identically satisfied, the necessary and sufficient conditions for the existence of a non-constant $b$ or $a$ are now clearly seen to be given by

$$
\begin{aligned}
& K S_{1}: \exists \text { non-constant } b \Longleftrightarrow \phi+\bar{\phi}=\phi^{\prime}+\bar{\phi}^{\prime}=0, \\
& K S_{2}: \exists \text { non-constant } a \Longleftrightarrow \phi+\bar{\phi}^{\prime}=0 .
\end{aligned}
$$

The corresponding space-times will henceforth be called $K S_{1}$ or $K S_{2}$ respectively. While the set $K S_{1} \cap K S_{2}$ has been dealt with in [14] (both conditions tacitly being assumed to be valid), it is at present not known whether or not $K S_{1} \cup K S_{2}$ yields the full set of KS space-times. The purpose of the present paper is restricted to constructing all space-times in which only one of the two conditions is satisfied. As a consequence these space-times should belong to the sub-classes with one constant eigenvalue of the HauserMalhiot CKT space-times [16]. Actually, as it was proven in [3, 11] that every type D space-time admitting an aligned conformal Killing tensor of Segre type [(11),(11)] is necessarily CKY, it follows that $\left(K S_{1} \backslash K S_{2}\right) \cup\left(K S_{2} \backslash K S_{1}\right)$ ought to be exactly the set of Hauser-Malhiot space-times with one constant eigenvalue. As the metrics found in paragraphs 3 and 4 below contain only one arbitrary function, this raises some doubts about the correctness of the results in [16].

Introducing 0 -weighted extension variables $U$ (real) and $V$ (complex), in accordance with (22), by

$$
\begin{aligned}
& \mathrm{\textrm { \rho }} \rho^{\prime}=\mathrm{\textrm {P }}^{\prime} \rho=-i U, \\
& \mathrm{P}^{\prime} \phi=\rho^{\prime}\left(2 \frac{\phi \phi^{\prime}}{|\tau|^{2}}+V\right)+i \frac{U \phi}{\rho},
\end{aligned}
$$

the Bianchi identities may be succinctly written in the following form:

$$
\begin{aligned}
& \mathrm{P} \phi=\frac{2 \rho}{|\tau|^{2}}\left(|\tau|^{4}-|\phi|^{2}\right), \\
& ð \phi=-\frac{1}{\tau^{\prime}}\left(2|\tau|^{4}+i \phi(U-H)-2 \phi^{2}\right), \\
& ð^{\prime} \phi=-2 \frac{\left|\phi^{2}\right|}{\tau}-\tau^{\prime}\left(2 \phi+2 \bar{\phi}+\rho \rho^{\prime}-2 v+\frac{i}{2 \rho} \mathrm{P} H\right), \\
& \mathrm{P} u=\rho(\phi-\bar{\phi}-3 i H), \\
& \precsim v=\frac{\rho \rho^{\prime}}{\tau^{\prime}}\left(\phi-\bar{\phi}^{\prime}\right)+i \tau(H+2 U), \\
& \precsim H=2 i \tau\left(|\tau|^{2}+2 u-4 \rho \rho^{\prime}\right)-2 i \frac{\rho \rho^{\prime}}{\tau^{\prime}}\left(2 \phi+2 \bar{\phi}^{\prime}+V\right) .
\end{aligned}
$$


Again these equations are accompanied by their primed versions, taking into account $U^{\prime}=U, V^{\prime}=V$ and $H^{\prime}=H$. Applying the commutators involving $\mathrm{P}^{\prime} \rho$ and using (42) yields two further relations, namely

$$
\begin{aligned}
& \mathrm{P} U=2 i \rho\left(\rho \rho^{\prime}+2 \phi+2 \bar{\phi}-2 v-2|\tau|^{2}\right), \\
& \precsim U=-2 i \frac{\rho \rho^{\prime}}{\tau^{\prime}}\left(2|\tau|^{2}+V\right) .
\end{aligned}
$$

Herewith all 'first level' integrability conditions on $\rho, \rho^{\prime}, \tau, \tau^{\prime}$ are identically satisfied. The next step is to construct the 'second level' integrability conditions by applying the commutators to $\phi, u, v, H$ and $U$.

When neither of the conditions $K S_{1}$ nor $K S_{2}$ hold, carrying out this procedure to the end results in a Pfaffian system for the involved variables, together with a set of algebraic relations among the latter. So far it has not been possible to complete the integrability analysis of this system. In the next two paragraphs all space-times will be constructed which belong to $K S_{1} \backslash K S_{2}$ or to $K S_{2} \backslash K S_{1}$.

\section{3. $K S_{1} \backslash K S_{2}$}

Consider space-times in which condition $K S_{1}$ holds, but not $K S_{2}$. A tedious but straightforward calculation, involving successive derivations of (40) and the 'second level' integrability conditions mentioned above, one obtains the following integrable system:

$$
\begin{aligned}
& \mathrm{P} \rho=0, \\
& \mathrm{P}^{\prime} \rho=\frac{2 \rho \rho^{\prime}}{|\tau|^{2}}\left(\phi+\phi^{\prime}\right) \\
& \text { ð } \rho=\frac{\rho}{\tau^{\prime}}\left(|\tau|^{2}-2 \phi\right) \\
& \mathrm{P} \tau=\frac{\rho}{\tau^{\prime}}\left(|\tau|^{2}-2 \phi\right), \\
& \widetilde{\partial} \tau=0, \\
& \check{\partial}^{\prime} \tau=\frac{2 \rho \rho^{\prime}}{|\tau|^{2}}\left(\phi+\phi^{\prime}\right)+i H, \\
& \mathrm{P} \phi=\frac{2 \rho}{|\tau|^{2}}\left(|\tau|^{4}+\phi^{2}\right), \\
& \mathrm{P}^{\prime} \phi=\frac{2 \rho^{\prime}}{|\tau|^{2}}\left(|\tau|^{4}-\phi^{2}+2 \phi \phi^{\prime}\right), \\
& \text { ð } \phi=\frac{1}{\tau^{\prime}}\left[i \phi H-2|\tau|^{4}+\frac{2 \rho \rho^{\prime}}{|\tau|^{2}}\left(\phi^{2}+\phi \phi^{\prime}\right)+2 \phi^{2}\right], \\
& \varlimsup^{\prime} \phi=\frac{1}{\tau}\left[i \phi H-2|\tau|^{4}+\frac{2 \rho \rho^{\prime}}{|\tau|^{2}}\left(\phi^{2}+\phi \phi^{\prime}\right)+2 \phi^{2}\right], \\
& \mathrm{P} H=\frac{2 \rho}{|\tau|^{2}}\left[2 i \rho \rho^{\prime}\left(2|\tau|^{2}+\phi \phi^{\prime}-\phi^{2}\right)+\phi H+4 i|\tau|^{4}\right], \\
& ð H=-8 i \tau\left(|\tau|^{2}+\rho \rho^{\prime}\right)-4 i \frac{\rho \rho^{\prime}}{\tau^{\prime}}\left(\phi-\phi^{\prime}\right)-6 i \tau E,
\end{aligned}
$$




$$
\begin{aligned}
& \mathrm{P} E=2 i \rho H-8 \rho \phi-4 \frac{\rho^{2} \rho^{\prime}}{|\tau|^{2}}\left(\phi+\phi^{\prime}\right), \\
& \left(\tau ð^{\prime}-\tau^{\prime} \text { ð }\right) E=4 \frac{\rho^{2} \rho^{\prime 2}}{|\tau|^{4}}\left(\phi^{2}-\phi^{\prime 2}\right)+2 i \frac{\rho \rho^{\prime}}{|\tau|^{2}} H\left(\phi-\phi^{\prime}\right) .
\end{aligned}
$$

Introducing 0 -weighted real variables $r$ and $m$ by

$$
r^{2}=Q \rho \rho^{\prime}, m=|\tau| \quad(Q= \pm 1)
$$

and writing $\phi=m^{2} r^{-2} \phi_{0}, \phi^{\prime}=m^{2} r^{-2} \chi_{0}$, one sees that $\phi_{0}, \chi_{0}$ are (imaginary) functions of $r$ given by

$$
\frac{\mathrm{d} \phi_{0}}{\mathrm{~d} r}=\frac{\mathrm{d} \chi_{0}}{\mathrm{~d} r}=2 \frac{\phi_{0} \chi_{0}+r^{4}}{r\left(\phi_{0}+\chi_{0}\right)}
$$

while (52-53) yield the exterior derivatives of $r$ and $m$ as

$$
\begin{aligned}
& \mathrm{d} r=\left(\phi_{0}+\chi_{0}\right)\left[\frac{m}{r}\left(\frac{\tau}{m} \boldsymbol{\omega}^{1}-\frac{m}{\tau} \boldsymbol{\omega}^{2}\right)+Q \frac{r}{\rho} \boldsymbol{\omega}^{3}+\frac{\rho}{r} \boldsymbol{\omega}^{4}\right], \\
& \mathrm{d} m=\frac{i}{2}\left(2 i Q\left(\phi_{0}+\chi_{0}\right)-H\right)\left[\frac{\tau}{m} \boldsymbol{\omega}^{1}-\frac{m}{\tau} \boldsymbol{\omega}^{2}\right]+\frac{2 m}{r}\left[Q \frac{r}{\rho} \chi_{0} \boldsymbol{\omega}^{3}+\frac{\rho}{r} \phi_{0} \boldsymbol{\omega}^{4}\right] .
\end{aligned}
$$

Note that the denominator in (60) is $\neq 0$, as otherwise $\phi_{0}^{2}=r^{4}$ which is in contradiction with $\phi_{0}$ being imaginary. Integrating (60) yields

$$
\begin{aligned}
& \chi_{0}=\phi_{0}-2 i c_{1}, \\
& \left(r^{2}-c_{2}\right)^{2}-\left(\phi_{0}-i c_{1}\right)^{2}=c_{1}^{2}+c_{2}^{2},
\end{aligned}
$$

$c_{1}, c_{2}$ real constants with $c_{1} \neq 0$ and $\left|r^{2}-c_{2}\right|<\sqrt{c_{1}^{2}+c_{2}^{2}}$ in order to have $\phi, \phi^{\prime}$ imaginary. By $(16,37)$ one has

$$
\mathrm{d} b=i\left(\frac{\tau}{m} \boldsymbol{\omega}^{1}-\frac{m}{\tau} \boldsymbol{\omega}^{2}\right) B .
$$

and hence

$$
\mathrm{d} r \wedge \mathrm{d} m \wedge \mathrm{d} b=2 i B Q \frac{m\left(\phi_{0}^{2}-\chi_{0}^{2}\right)}{r}\left(\frac{\tau}{m} \boldsymbol{\omega}^{3} \wedge \boldsymbol{\omega}^{4} \wedge \boldsymbol{\omega}^{1}-\frac{m}{\tau} \boldsymbol{\omega}^{3} \wedge \boldsymbol{\omega}^{4} \wedge \boldsymbol{\omega}^{2}\right) \neq 0,
$$

showing that one can use $r, m$ and $b$ as coordinates. As $B$ satisfies the same equations $(16,37)$ as $b$, it follows that $B=B(b)$, allowing one to redefine $b$ such that $B=1$. Defining a fourth coordinate $x$ by

$$
\frac{1}{2}\left(\frac{\tau}{m} \boldsymbol{\omega}^{1}+\frac{m}{\tau} \boldsymbol{\omega}^{2}\right)=h_{1} \mathrm{~d} b+h_{2} \mathrm{~d} r+h_{3} \mathrm{~d} m+\mathrm{d} x,
$$

the equations $(55,56)$ imply

$$
\begin{aligned}
\mathrm{d} H= & \Sigma_{1}(b, m, r) \mathrm{d} b+\left(16 Q c_{1} m h_{2}+\Sigma_{2}(b, m, r)\right) \mathrm{d} r \\
& -\left(16 Q c_{1} m h_{3}-\frac{H-4 Q\left(c_{1}+i \phi_{0}\right)}{m}\right) \mathrm{d} m-16 Q c_{1} m \mathrm{~d} x,
\end{aligned}
$$

showing that

$$
H=-16 Q c_{1} m x+2 i Q\left(\phi_{0}+\chi_{0}\right)+m H_{0},
$$

with $H_{0}=H_{0}(b, r, m)$. It also follows that $h_{2}$ is a freely specifiable function of $b, r$ and $m$ (using a translation of $x$ ). Choosing $h_{2}=-m r /\left[4 Q c_{1}\left(c_{1}+i \phi_{0}\right)\right]$, (68) shows that 
$H_{0}=H_{0}(b, m)$, such that $h_{3}$ can be made 0 by a further $(b, m)$-dependent translation of $x$. Herewith (68) eventually becomes

$$
\mathrm{d} H_{0}+u_{0} \mathrm{~d} b=0
$$

with

$$
u_{0}=16 Q c_{1} h_{1}-4 u-\frac{1}{2} H_{0}^{2}+16 Q c_{1} x H_{0}+6 m^{2}-128 c_{1}^{2} x^{2}+12 Q r^{2},
$$

showing that $H_{0}=H_{0}(b)$ and $u_{0}=u_{0}(b)$. It remains to determine the function $E$ (or $u$ ) from $(57,58)$ in order to obtain $h_{1}$ from $(71)$. One readily finds

$$
h_{1}=\frac{x H_{0}}{2}-Q\left(4 c_{1} x^{2}+\frac{m^{2}}{4 c_{1}}\right)+F
$$

where $F=F(b)$ can be made 0 by a $b$-dependent translation of $x$ and a corresponding re-definition of $H_{0}$. This gives the following expression for the dual basis $\left(\rho, \phi_{0}\right.$ and $\chi_{0}$ being imaginary):

$$
\begin{aligned}
& \frac{\tau}{m} \boldsymbol{\omega}^{1}=\mathrm{d} x+\frac{i Q m r}{4 c_{1}\left(\phi_{0}-i c_{1}\right)} \mathrm{d} r+\left(\frac{H_{0} x}{2}-4 Q c_{1} x^{2}-\frac{2 i c_{1}+Q m^{2}}{4 c_{1}}\right) \mathrm{d} b, \\
& \frac{1}{\rho} \boldsymbol{\omega}^{3}=\frac{i Q}{4 c_{1}}\left(\frac{\mathrm{d} m}{m}-\frac{\phi_{0}}{\phi_{0}-i c_{1}} \frac{\mathrm{d} r}{r}\right)+i\left(Q \frac{r^{2} H_{0}-4 m i \phi_{0}}{8 r^{2} c_{1}}-2 x\right) \mathrm{d} b, \\
& \rho \boldsymbol{\omega}^{4}=\frac{i r^{2}}{4 c_{1}}\left(-\frac{\mathrm{d} m}{m}-\frac{\chi_{0}}{\chi_{0}+i c_{1}} \frac{\mathrm{d} r}{r}\right)-i\left[\frac{r^{2} H_{0}-4 m i \phi_{0}}{8 c_{1}}-m-2 Q r^{2} x\right] \mathrm{d} b,
\end{aligned}
$$

implying

$$
\begin{aligned}
& R=-12\left(Q r^{2}+m^{2}+E\right) \\
& E=-2 m^{2}-\frac{8}{3} Q r^{2}+32 c_{1}^{2} x^{2}+\frac{1}{12} H_{0}^{2}-\frac{1}{6} \frac{d H_{0}}{d b}-4 Q c_{1} x H_{0}-\frac{4 Q}{3 r^{2}} \phi_{0} \chi_{0}, \\
& H=\left(H_{0}-16 Q c_{1} x\right) m+2 i Q\left(\phi_{0}+\chi_{0}\right), \\
& \Phi_{00}=-\rho^{2} \\
& \Phi_{22}=-\frac{r^{4}}{\rho^{2}} \\
& \Phi_{11}=-\frac{9}{2} Q r^{2}-\frac{7}{2} m^{2}-\frac{3}{2} E-\frac{4 Q \phi_{0} \chi_{0}}{r^{2}} \\
& \Phi_{01}=\frac{\rho \tau}{r^{2}}\left(2 \phi_{0}-3 r^{2}\right), \\
& \Phi_{12}=-\frac{Q \tau}{\rho}\left(2 \chi_{0}+3 r^{2}\right) \\
& \Phi_{02}=-\tau^{2} .
\end{aligned}
$$

\section{4. $K S_{2} \backslash K S_{1}$}

The analysis of the space-times belonging to $K S_{2} \backslash K S_{1}$ proceeds along the same lines, but is greatly facilitated by noticing that the conditions $(16,17)$ are transformed into each other under the Sachs [13] asterisk operation $\mathrm{P}^{*}=ð, \mathrm{P}^{*}=-\varlimsup^{\prime}, ð^{*}=-\mathrm{P}, \varlimsup^{* *}=\mathrm{P}^{\prime}$. The same of course holds for the pairs of integrability conditions $(36,37),(38,39),(40,41)$. 
This becomes obvious after writing down the Sachs-transforms of $\phi, \bar{\phi}$ and $\phi^{\prime}$ (recall that complex conjugation and the Sachs-operation do not commute):

$$
\phi^{*}=\frac{\rho \rho^{\prime}}{|\tau|^{2}} \phi, \quad \phi^{*}=\frac{\rho \rho^{\prime}}{|\tau|^{2}} \phi^{\prime}, \quad \bar{\phi}^{*}=\frac{\rho \rho^{\prime}}{|\tau|^{2}} \bar{\phi}^{\prime}, \quad \bar{\phi}^{*}=\frac{\rho \rho^{\prime}}{|\tau|^{2}} \bar{\phi} .
$$

Defining coordinates $r$ and $m$ as in (59) one now finds $\phi=\phi(m)$, with

$$
\phi+\bar{\phi}=2 c_{1} \neq 0
$$

and

$$
\left(\phi-c_{1}\right)^{2}-\left(m^{2}-c_{2}\right)^{2}=c_{1}^{2}-c_{2}^{2} .
$$

Here $c_{1}, c_{2}$ are real constants and $\left|m^{2}-c_{2}\right|<\sqrt{c_{2}^{2}-c_{1}^{2}}$ in order to obtain (86). Without further ado the results are presented below ( $\rho$ and $\phi-c_{1}$ being imaginary):

$$
\begin{aligned}
\tau \boldsymbol{\omega}^{1} & =\frac{m^{2}}{4 c_{1}}\left(\frac{\mathrm{d} r}{r}-\frac{\phi-2 c_{1}}{\phi-c_{1}} \frac{\mathrm{d} m}{m}\right)+\left[-Q \frac{4 i Q r \phi-m^{2} H_{0}}{8 c_{1}}+i\left(r+2 i Q m^{2} x\right)\right] \mathrm{d} a, \\
\frac{r}{\rho} \boldsymbol{\omega}^{3} & =-i Q \mathrm{~d} x+\frac{m r}{4 c_{1}\left(\phi-c_{1}\right)} \mathrm{d} m-i\left(\frac{H_{0} x}{2}-4 c_{1} x^{2}-\frac{r^{2}}{4 c_{1}}+Q\right) \mathrm{d} a, \\
\frac{\rho}{r} \boldsymbol{\omega}^{4} & =i \mathrm{~d} x-Q \frac{m r}{4 c_{1}\left(\phi-c_{1}\right)} \mathrm{d} m+i\left(\frac{H_{0} x}{2}-4 c_{1} x^{2}-\frac{r^{2}}{4 c_{1}}\right) \mathrm{d} a,
\end{aligned}
$$

implying

$$
\begin{aligned}
& E=-2 Q r^{2}-\frac{8}{3} m^{2}+32 Q c_{1}^{2} x^{2}+\frac{Q}{12} H_{0}^{2}-\frac{1}{6} \frac{d H_{0}}{d a}-4 Q c_{1} x H_{0}+\frac{4 Q}{3 m^{2}} \phi \bar{\phi} \\
& H=\left(H_{0}-16 c_{1} x\right) r+4 i\left(\phi-c_{1}\right) \\
& \Phi_{11}=\frac{9}{2} m^{2}+\frac{7}{2} Q r^{2}+\frac{3}{2} E-\frac{4 \phi \bar{\phi}}{m^{2}} \\
& \Phi_{01}=\frac{\rho \tau}{m^{2}}\left(2 \phi-3 m^{2}\right) \\
& \Phi_{12}=-\frac{Q r^{2} \tau}{m^{2} \rho}\left(2 \phi+3 m^{2}\right)
\end{aligned}
$$

with the expressions for $R, \Phi_{00}, \Phi_{22}$ and $\Phi_{02}$ being identical to those of the previous paragraph.

As the Sachs transform destroys the reality conditions on the null-tetrad, it is to be expected that the two solution families will be related by a complex coordinate transformation, in combination with a possible complex transformation of the parameters and the free functions. Indeed such a transformation exists and one can verify that the $K S_{1}$ line-elements are obtained by applying the following operations to $K S_{2}$ :

$$
\begin{aligned}
& r \rightarrow m Q^{-1 / 2}, m \rightarrow r q^{1 / 2}, a \rightarrow b Q^{1 / 2}, x \rightarrow-i Q^{1 / 2} x+g \\
& c_{1} \rightarrow i Q c_{1}, \phi \rightarrow q \phi_{0}, h_{0} \rightarrow Q^{1 / 2} h_{0}-16 q c_{1} g
\end{aligned}
$$

with $g(b)$ a solution of

$$
2 g^{\prime}+Q^{1 / 2}-i h_{0} g+\frac{8 i c_{1}}{q} Q^{-1 / 2} g^{2}=0
$$




\section{Summary}

All KS space-times are constructed in which one of the integrability conditions $(40,41)$ (both of which were assumed to be valid in [14]) are violated. This leads to the conformal classes of $K S_{1}$ and $K S_{2}$ space-times discussed in paragraphs 3 and 4 . Each of these classes is characterized by a single arbitrary function $\left(H_{0}\right)$. In general none

of the corresponding space-times admits any isometries (when $H_{0}$ is constant then a one-dimensional group of isometries exists), but has a conformal representant $(\mathcal{M}, \hat{g})$, admitting a Killing tensor with precisely one constant eigenvalue. The explicit form of the resulting space-times raises doubts about the correctness of the Hauser-Malhiot metrics [16] admitting a conformal Killing tensor with one constant eigenvalue. It is an open problem whether the classes $K S_{1}$ and $K S_{2}$, together with the metrics presented in [14] and [20] exhaust the full set of KS space-times. There is also no guarantee that the classes $K S_{1}$ and $K S_{2}$ discussed in the present paper have a conformal representant possessing any physically interesting interpretation. These issues will be dealt with in a forthcoming publication.

\section{Acknowledgment}

I thank Brian Edgar (Linköping University) for his comments on an earlier version of this paper.

\section{References}

[1] I.N. Glass and J. Kress, 1999 J. Math. Phys. 40, 309

[2] B. Carter and R.G. McLenaghan, 1979 Phys. Rev. D 19, 1093

[3] B. Coll, J.J. Ferrando and J.A. Saez, 2006 J. Math. Phys. 47, 062503

[4] C.D. Collinson, 1976 Int. J. Theor. Phys. 15, 311

[5] S.R. Czapor and R.G. McLenaghan, 1982 J. Math. Phys. 23, 2159

[6] R. Debever and R.G. McLenaghan, 1981 J. Math. Phys. 22, 1711

[7] W. Dietz and W. Rüdiger, 1980 Proc. R. Soc. A 375, 361

[8] W. Dietz and W. Rüdiger, 1981 Proc. R. Soc. A 381, 315

[9] W. Dietz and W. Rüdiger, 1980 Gen. Rel. Grav. 12, 545

[10] J.J. Ferrando and J.A. Saez, 2003 Gen. Rel. Grav. 35, 7

[11] J.J. Ferrando and J.A. Saez, 2007 Gen. Rel. Grav. 39, 343

[12] R. Floyd The dynamics of Kerr fields, PhD Thesis, University of London, 1973

[13] R. Geroch, A. Held and R. Penrose, 1973 J. Math. Phys. 14, 874

[14] B.P. Jeffryes, 1984 Proc. R. Soc. A 392, 323

[15] I. Hauser and R.J. Malhiot, 1976 J. Math. Phys. 17, 1306

[16] I. Hauser and R.J. Malhiot, 1978 J. Math. Phys. 19, 187

[17] L. Hughston and P. Sommers, 1973 Comm. Math. Phys. 32, 147

[18] N. Kamran and R.G. McLenaghan, 1984 Phys. Rev. D 30, 357

[19] R.G. McLenaghan and P.L. Spindel, 1979 Phys. Rev. D 20, 409

[20] R.G. McLenaghan and N. Van den Bergh, 1993 Class. Quantum Grav. 10, 2179

[21] D. Kramer, H. Stephani, M. A. H. MacCallum, C. Hoenselaers and E. Herlt, Exact solutions of Einstein's field equations, Cambridge University Press, 2003

[22] E. T. Newman and R. Penrose, 1962 J. Math. Phys. 3, 566 
[23] T. Papacostas, 1983 Bull. Cl. Sciences, Acad. R. Belgique LXIX, 495

[24] T. Papacostas, 1983 Bull. Cl. Sciences, Acad. R. Belgique LXIX, 641

[25] T. Papacostas, 1985 Gen. Rel. Grav. 17, 149

[26] R. Penrose, 1973 Ann. N.Y. Acad. Sci. 224, 125

[27] J. Plebañski and S. Hacyan, 1976 J. Math. Phys. 17, 2203

[28] H. Stephani, 1978 Gen. Rel. Grav. 9, 789

[29] S. Tachibana, 1969 Tohoku Math. J. 21, 56

[30] K. Yano and S. Bochner, Curvature and Betti numbers, Ann. Math. Studies vol. 32, Princeton University Press, 1953

[31] M. Walker and R. Penrose, 1970 Comm. Math. Phys. 18, 265 